\title{
Ciclos econômicos na atividade industrial brasileira: uma análise no domínio do tempo e da frequência
}

Business cycles in the Brazilian industrial activity: an analysis on the time and frequency domain

\author{
Cristiano da Costa da Silva (1) \\ Nicolino Trompieri Neto(2) \\ Ivan Castelar ${ }^{(3)}$ \\ Erika Vanessa Alves da Silva(4)
}

\author{
(1) Universidade do Estado do Rio Grande do Norte \\ (2) Instituto de Pesquisa e Estratégia Econômica do Ceará \\ Universidade de Fortaleza \\ (3) Universidade Federal do Ceará \\ (4) Companhia Pernambucana de Saneamento
}

\begin{abstract}
This article analyzes the monthly behavior of the industrial production of the states of Bahia, Minas Gerais, Pernambuco, Paraná, Rio de Janeiro, Rio Grande do Sul and São Paulo, in the period from 2002:01 to 2014:12. To this, was used the methodology of common trends and cycles of Vahid and Engle (1993). The tests prove the existence of two common trends and five common cycles. Deviations from the long-term equilibrium in the industrial activity of São Paulo influence the production trajectory of the other states studied, while permanent shocks in Rio Grande do Sul affect only the industrial dynamics of Paraná. To analyze the business cycles, were introduced tools from the spectral analysis, thus obtaining additional information regarding the synchronization of the short-term dynamics. The hypothesis of co-movements between the Paraná and São Paulo cycles was confirmed through frequency domain analysis.
\end{abstract}

\section{Keywords}

industrial activity, spectral snalysis, so-movements, common cycles, common trends.

JEL Codes E31, E32, C23.

\section{Resumo}

Este artigo analisa o comportamento mensal da produção industrial dos estados da Bahia, Minas Gerais, Pernambuco, Paraná, Rio de Janeiro, Rio Grande do Sul e São Paulo, no periodo de 2002:01 até 2014:12, através da metodologia de tendências e ciclos comuns de Vahid e Engle (1993). Os testes comprovam a existência de duas tendências comuns e cinco ciclos comuns. Os resultados apontam que desvios do equilíbrio de longo prazo na atividade industrial de São Paulo influenciam a trajetória de produção dos demais estados estudados, ao passo que choques permanentes no Rio Grande do Sul afetam efetivamente apenas a dinâmica industrial do Paraná. Para efetuar a análise dos ciclos dos negócios foram implantadas ferramentas oriundas da análise espectral, obtendo, assim, informações adicionais com relação à sincronização das dinâmicas do curto prazo. A hipótese de comovimentos entre os ciclos do Paraná e São Paulo foi confirmada através da análise no domínio da frequência.

\section{Palavras-chave}

atividade industrial, análise espectral, comovimentos, ciclos comuns, tendências comuns.

Códigos JEL E31, E32, C23. 


\section{Introdução}

A adoção de políticas industriais por parte do setor público é considerada assunto controverso dentro da literatura econômica. Pois, apesar de seu genuíno objetivo de fomentar o crescimento/desenvolvimento econômico de uma determinada região ou de atenuar os efeitos de um choque estrutural exógeno, a aplicabilidade de políticas não horizontais - ao beneficiar um determinado um dado setor ou região - tem o potencial de acentuar as disparidades regionais existentes, dependendo do grau de dissimilaridades nos ciclos econômicos ${ }^{1}$ dos Estados.

Heterogeneidades na estrutura regional industrial podem contribuir para diferenças no comportamento dos ciclos econômicos, na medida em que diferentes composições de indústria tendem a experimentar diferentes choques estruturais, resultando em específicos ciclos econômicos. Nesse sentido, Monteiro e Lima (2017) evidenciam que os diferentes estágios de maturação e composição entre segmentos tradicionais e tecnológicos na indústria de transformação afetam a dinâmica de desindustrialização regional no Brasil.

Por outro lado, a integração entre regiões e o maior grau de comercialização induzem a propagação dos ciclos entre os estados de diferentes regiões. A existência de um comportamento similar entre a dinâmica de resposta dos estados a esses choques evidencia a presença de comovimentos entre eles. Saboia (2013) reporta uma dinâmica de desconcentração regional na indústria brasileira ao longo dos anos 2000, o que tende a reforçar o grau de comercialização entre as unidades federativas.

O objetivo deste artigo consiste em analisar os ciclos de negócios das atividades industriais de um conjunto de Estados do Brasil ${ }^{2}$ no domínio do tempo e da frequência. A proposta de investigar o comportamento da produção industrial com ênfase sobre um horizonte curto de tempo - ciclos econômicos - é justificada principalmente por duas razões:

a) Um maior entendimento das relações entre as flutuações de curto prazo das atividades industriais dos estados do Brasil pode contribuir para

1 A literatura retrata ciclos de negócios como flutuações na atividade econômica em torno de uma tendência estocástica que formam um padrão regular, definido em termos de expansão ou recessão.

2 Os estados selecionados foram Bahia, Minas Gerais, Paraná, Pernambuco, Rio de Janeiro, Rio Grande do Sul e São Paulo. 
a implantação de políticas públicas mais adequadas ao setor industrial por parte do governo;

b) Diante de um ambiente de incerteza os agentes atribuem maior peso ao curto prazo em seu processo decisório, tornando a volatilidade do produto industrial um fator importante para entender o ciclo de negócio (Issler; Vahid, 2001; Engle; Kozicki, 1993).

Seguindo a metodologia de Vahid e Engle (1993) será investigado se a atividade industrial dos principais estados das regiões Sul, Sudeste e Nordeste do Brasil apresentam dinâmicas comuns de curto e longo prazo, através da decomposição das séries em componente de tendência e componente de ciclo.

Por fim, cabe destacar que grande parte da literatura especializada na análise de tendências e ciclos comuns tem concentrado atenção em ferramentas como: a análise da correlação linear entre as flutuações de curto prazo; a verificação da existência de precedência temporal entre os ciclos dos negócios e; o efeito de longo prazo no conjunto de variáveis de um choque permanente sobre uma série em questão como principais indicadores para investigar o grau de associação entre os movimentos de curto e longo prazos das séries econômicas decompostas, o que denota o caráter estático da análise de ciclos econômicos.

Para superar essa lacuna o presente trabalho segue a linha de Gutierrez e Gomes (2009), lançando mão da análise espectral para investigar o grau de associação dos ciclos econômicos das atividades industriais em diferentes frequências ou diferentes componentes cíclicos ao longo do intervalo amostral, através das ferramentas de coerência, coincidência e fase.

Com o intuito de atingir os objetivos propostos o artigo foi particionado em cinco seções: além desta parte introdutória, haverá a posteriori a revisão de literatura. $\mathrm{Na}$ seção seguinte serão apresentadas as técnicas econométricas necessárias para o alcance do objetivo proposto. A quarta seção destina-se à apresentação dos resultados obtidos e da base de dados, enquanto a última seção traz as considerações finais.

\section{Referencial teórico}

Atualmente existe ampla discussão sobre análise de séries macroeconômicas em termos de componentes de curto prazo e longo prazo. Em 
termos econométricos, existem diversas estratégias de decomposição, a lista estende-se de modelos univariados, como o filtro Hodrick e Prescott (1981), Baxter e King (1999), e de modelos estruturais de estado-espaço estimados via filtro de Kalman, até modelos multivariados, entre os quais se destacam a decomposição estrutural de Gonzalo e Granger (1995) e a decomposição de Beveridge-Nelson-Stock-Watson (Vahid; Engle, 1993), o qual é utilizado no presente artigo.

Essas técnicas, basicamente, diferem-se com relação às hipóteses levantadas acerca do comportamento da economia, como o processo gerador da trajetória de crescimento das séries, definição do intervalo dos ciclos econômicos, definição determinística ou estocástica da tendência de longo prazo. Diferenças estas que repercutem sobre as conclusões gerais encontradas referentes à dinâmica de curto, médio e longo prazos das economias, sendo, portanto, a razoabilidade das hipóteses fator-chave para a análise acurada da trajetória econômica de um setor/região (Wang, 2010).

A abordagem de Vahid e Engle revisa as especificações de cointegração e correlação serial para um quadro estrutural de séries de tempo, de modo a testar a presença de ciclos comuns entre as variáveis agregadas. Issler e Vahid (2001) destacam a importância da utilização da abordagem multivariada supracitada na presença de restrição de comovimentos de curto prazo entre as variáveis analisadas, na medida em que a existência de ciclos comuns entre as variáveis afeta de maneira efetiva a importância relativa dos componentes de curto prazo nas flutuações das variáveis em nível.

Outra questão técnica refere-se ao nível de desagregação ideal dos dados na análise dos comovimentos entre setores/regiões. Engle (1984) e Long e Plosser (1987) destacam que a utilização de dados agregados pode implicar superestimação das inter-relações entre os ciclos econômicos de diferentes setores/regiões, gerando correlações espúrias e levando a conclusões viesadas.

Entre os estudos que se enquadram nas duas linhas de pesquisa abordadas, destacam-se os artigos de Carlino e Sill (2001), Mejia-Reyes, Gómez e Balboa (2004), Wakerly, Scott e Nason (2006), Gutierrez e Gomes (2009) e Wang (2013).

Carlino e Sill (2001) utilizaram especificações de Beveridge e Nelson (1981) e de Gonzalo e Granger (1995) no intuito de investigar os componentes de curto e longo prazo da renda per capita das regiões dos Estados Unidos. Os resultados obtidos a partir da decomposição de Gonzalo 
e Granger (1995) indicam a presença de ciclos comuns entre as regiões, existindo, no entanto, forte variabilidade quanto ao nível de volatilidade dos ciclos regionais.

Ao investigarem a natureza dos ciclos econômicos nos setores industriais da economia mexicana a partir da concepção clássica de ciclos de negócios, Mejia-Reyes, Gómez e Balboa (2004) encontraram padrões assimétricos no comportamento dos ciclos de negócios da atividade industrial do México, observando que as taxas de crescimento das atividades produtivas industriais durante os períodos de expansão apresentaram-se superiores às taxas de queda na atividade em períodos de recessão.

Wakerly, Scott e Nason (2006) utilizam a especificação de Vetores Autorregressivos (VAR), com restrições de curto e longo prazos, proposta por Vahid e Engle (1993,) na análise das flutuações temporais do Produto Interno Bruto (PIB) per capita do Canadá, em nível regional. ${ }^{3}$ Os resultados apontam a presença de duas tendências comuns divergentes, refutando a hipótese de convergência entre as regiões.

A partir da decomposição multivariada de Beveridge-Nelson-Stock-Watson, Gutierrez e Gomes (2009) investigam a natureza dos ciclos de negócios dos países membros do Mercosul, entre o período de 1951 a 2003. O estudo utiliza ferramentas do domínio da frequência - coerência e diferença de fase - para investigar a hipótese de comovimentos entre os ciclos econômicos dos países. A análise espectral confirmou a presença de sincronização entre dois subgrupos - Brasil e Argentina; Paraguai e Uruguai.

Wang (2013) analisa os comovimentos entre os componentes transitórios - ciclos - dos setores produtivos do Reino Unido no domínio do tempo e da frequência. Os resultados apontam que os setores possuem comportamento similar em baixas frequências (ciclos de longo prazo), mas, por outro lado, apresentam comportamentos heterogêneos em ciclos de média e alta frequência (médio e curto prazos).

Do ponto de vista nacional, a partir do teste de causalidade no domínio da frequência desenvolvido por Breitung e Candelon (2006), Rocha e Souza (2018) reportam que o crédito ao setor privado exerce causalidade sobre o crescimento econômico brasileiro no horizonte de longo prazo.

3 As regiões em análise foram British Columbia, Ontario, Quebec, Prairies (região que agrupa Alberta, Manitoba e Saskatchewan) e Maritimes (referindo-se à Newfoundland, New Brunswick, Nova Scotia e Prince Edward Island). 
Realizada esta discussão, o presente artigo contribui para a literatura ao modelar os ciclos econômicos da atividade industrial do Brasil em um ambiente multivariado, fazendo-se valer de uma base de dados desagregada em nível de unidades federativas.

\section{Metodologia}

\subsection{Teste de raiz unitária}

Para verificar a hipótese de que o índice de produção industrial para os Estados do Brasil são séries temporais que oscilam em torno de uma tendência estocástica, o aspecto metodológico inicial consiste em implementar os testes de estacionariedade.

Devido à provável existência de quebra na tendência observada nos dados, faremos uso de quatro testes para verificar a ocorrência de raiz unitária com quebra estrutural. Os testes aplicados para averiguar a existência de raiz unitária com quebra estrutural são os testes propostos por Zivot e Andrews (1992), Perron (1997), Lanne, Lütkepohl e Saikkonen (2002) e uma modificação do teste ADF permitindo uma única quebra estrutural, formulado a partir dos trabalhos de Perron (1989), Perron e Vogelsang (1992), Vogelsang e Perron (1998) e Banerjee, Lumsdaine e Stock (1992). Todos os testes de quebra estrutural utilizados aqui permitem que a determinação do ponto de quebra estrutural seja realizada de forma endógena, a partir das informações expostas nos dados.

\subsection{Modelo econométrico}

A decomposição multivariada de Beveridge-Nelson-Stock-Watson (BNSW), apresentada em Vahid e Engle (1993), testa a existência de dinâmicas comuns de longo e curto prazos sobre um modelo VAR estimado. Inicialmente, considere os índices de produção industrial dos $n$ Estados, representados por um Vetor Auto Regressivo (VAR) de ordem finita $p$.

$$
Y_{t}=\phi_{1} Y_{t-1}+\phi_{2} Y_{t-2}+\ldots+\phi_{p} Y_{t-p}+\varepsilon_{t}
$$


onde $Y_{t}$ é um vetor de $n$ séries integradas de primeira ordem, $I(1)$, e $\phi_{i}$, $i=1,2, \ldots, p$ são matrizes de dimensão $n \times n$ e $\varepsilon_{t} \sim \operatorname{Normal}(0, \Omega), E\left(\varepsilon_{t}\right)=0$ e $E\left(\varepsilon_{t} \varepsilon_{\tau}\right)=\left\{\Omega\right.$, se $\mathrm{t}=\tau$ e $0_{n \times n}$, se $\left.\mathrm{t} \neq \tau\right\}$ é não singular. Adicionalmente, o modelo (1); onde $\Omega$ pode ser escrito equivalentemente como:

$$
\Pi\left(L_{t}\right) Y_{t}=\varepsilon_{t}
$$

onde $\Pi\left(L_{t}\right)=I_{n}-\sum_{i=1}^{p} \phi_{\mathrm{i}} L^{i}$, e $L$ representa o operador defasagem. Note que se $L=1$, então $\Pi(1)=I_{n}-\sum_{i=1}^{p} \phi_{\mathrm{i}}$.

\subsection{Restrições de longo prazo - cointegração}

Assuma as seguintes hipóteses:

\section{Proposição 1}

A matriz $\Pi($.$) satisfaz:$

1) Posto $(\Pi(1))=r, 0<r<n$, tal que $\Pi$ (1) pode ser expresso como $\Pi(1)=-\alpha \beta^{\prime}$, onde $\alpha$ e $\beta$ são matrizes (nxr) com posto cheio $r$.

2) A equação característica $|\Pi(L)|=0$ possui $n-r$ raízes iguais a 1 e todas as outras fora do circulo unitário.

A hipótese 1 implica que $Y_{t}$ é uma variável cointegrada de ordem $(1,1)$. Os elementos de $\alpha$ representam os coeficientes de ajuste de velocidade ao equilíbrio de longo prazo e $\beta$ o espaço de cointegração. Decompondo a matriz polinomial $\Pi(L)=\Pi(1) L+\Pi^{*}(L) \Delta$, onde $\Delta \equiv(1-L)$ denota o operador diferença, pode-se obter um modelo de vetor de correção de erros (VEC):

$$
\Delta Y_{t}=\alpha \beta^{\prime} Y_{t-1}+\sum_{i=1}^{p} \Gamma_{j} \Delta Y_{t-j}+\varepsilon_{t}
$$

onde $\alpha \beta^{\prime}=-\Pi(1), \Gamma_{j}=-\sum_{k=j+1}^{p} \phi_{k}(j=1,2, \ldots, p-1)$ e $\Gamma_{0}=\mathrm{I}_{n}$. 
Será aplicado o teste desenvolvido por Johansen, Mosconi e Nielsen (2000), onde a distribuição assintótica difere do usual "teste do traço" de Johansen (1988) por utilizar variáveis dummies que identificam quebras estruturais na tendência dos vetores cointegrantes.

\subsection{Restrições de curto prazo}

\section{Definição 1}

A equação (3) apresentará característica de correlação serial comum (SCCF) se existir uma matriz $\widehat{\beta}_{n \times s}$, de posto $s$, representando o espaço de cocaracterização, tal que $\tilde{\beta}^{\prime} \Delta Y_{t}=\tilde{\beta}^{\prime} \varepsilon_{t}$ onde $\tilde{\beta}^{\prime} \varepsilon_{t}$ é um vetor de dimensão $s$ que é uma inovação em relação a toda informação anterior ao período $t$.

Consequentemente existirão restrições de características de correlação serial comum se as seguintes condições forem satisfeitas:

Proposição 2

$$
\tilde{\beta}^{\prime} \Gamma_{j}=0_{s \times n} \quad \forall i=1, \ldots, p-1
$$

Proposição 3

$$
\tilde{\alpha}^{\prime} \beta \alpha^{\prime}=0_{s x n}
$$

\subsection{Decomposição tendência-ciclo}

A decomposição tendência-ciclo BNSW pode ser introduzida por meio da representação de Wold do vetor estacionário $\Delta Y_{t}$ dada por:

$$
\Delta Y_{t}=\mathrm{C}(\mathrm{L}) \varepsilon_{t}
$$

onde $\mathrm{C}(\mathrm{L})=\sum_{i=0}^{\infty} C_{i} L^{i}$ é uma matriz polinomial no operador defasagem, $C_{0}=I_{n}$ e $\sum_{i=0}^{\infty} i\left|C_{i}\right|<\infty \quad$ Usando a seguinte fatoração polinomial $C(L)=$ 
$=C(1)+\Delta C^{*}(L)$, é possível decompor $\Delta Y_{t}$ como:

$$
\Delta Y_{t}=\mathrm{C}(1) \varepsilon_{t}+\Delta \mathrm{C}^{*}(L) \varepsilon_{t}
$$

onde $C_{i}^{*}=\sum_{j>i}^{\infty}\left(-C_{j}\right), i \geq 0$, e $C_{0}^{*}=I_{n}-C(1)$. Ignorando os valores iniciais $Y_{0}$ e integrando ambos os lados de (5), obtém-se:

$$
Y_{t}=\mathrm{C}(1) \sum_{j=1}^{T} \varepsilon_{t}+\mathrm{C}^{*}(L) \varepsilon_{t}=T_{t}+C_{t}
$$

A equação (6) representa a decomposição BNSW onde $Y_{t}$ é decomposto em $n$ processos de passeio aleatório - tendências estocásticas - e $n$ processos estacionários - ciclos. Assim, $T_{t}=\mathrm{C}(1) \sum_{j=1}^{T} \varepsilon_{t}$ e $C_{t}=\mathrm{C}^{*}(L) \varepsilon_{t}$ representam o componente de tendência e ciclo, respectivamente. Se as restrições de longo prazo forem verificadas, então os $r$ vetores de cointegração eliminam o componente de tendência estocástica, implicando que $\beta^{\prime} \mathrm{C}(1)=0$. Desse modo C1 tem dimensão $n-r$, o que implica a existência de $n-r$ tendências comuns. Analogamente, sob as restrições de curto prazo, existem $s$ vetores de cocaracterização que eliminam os ciclos, $\tilde{\beta}^{\prime} C^{*}(L)=0$, e $C^{*}(L)$ possui dimensão $n-s$, o que é o número de ciclos comuns.

Uma vez que os vetores de cointegração e cocaracterização são linearmente independentes (Vahid e Engle, 1993), o número de vetores de cointegração adicionado ao de vetores de cocaracterização deve ser menor ou igual ao número de variáveis $-r+s \leq n$.

Para obter as tendências comuns basta pré-multiplicar a equação (6) por $\tilde{\beta}$, de tal forma:

$$
\tilde{\beta}^{\prime} Y_{t}=\tilde{\beta}^{\prime} \mathrm{C}(1) \sum_{j=1}^{T} \varepsilon_{t}=\tilde{\beta}^{\prime} T_{t}
$$

Essa combinação linear não contem ciclos porque os vetores cocaracterísticos os eliminam. Da mesma forma, para obter os ciclos comuns é necessário e suficiente pré-multiplicar a equação (6) por $\tilde{\beta}^{\prime}$ :

$$
\beta^{\prime} Y_{t}=\beta^{\prime} \mathrm{C}(1) \mathrm{C}^{*}(L) \varepsilon_{t}=\beta^{\prime} C_{t}
$$

Essa combinação linear não contém componentes de tendência, porque estas são eliminadas pelos vetores de cointegração. Um caso especial 
emerge quando $r+s=n$. Nesse caso, é extremamente simples estimar os componentes de tendência e ciclo de $Y_{t}$. Uma vez que $\tilde{\beta}^{\prime}$ e $\beta^{\prime}$ são matrizes linearmente independentes, pode-se construir uma matriz $A$, tal que $A_{n x n}=\left(\tilde{\beta}^{\prime}, \beta^{\prime}\right)^{\prime}$ possui posto completo, sendo invertível. Note que a matriz inversa pode ser particionada como $A^{-1}=\left(\tilde{\beta}^{-} \beta^{-}\right)$e os componentes de tendência e ciclo podem ser obtidos como a seguir:

$$
Y_{t}=A^{-1} A Y_{t}=\tilde{\beta}^{-}\left(\tilde{\beta}^{\prime} Y_{t}\right)+\beta^{-}\left(\beta^{\prime} Y_{t}\right)=T_{t}+C_{t}
$$

onde $T_{t}=\tilde{\beta}^{-}\left(\tilde{\beta}^{\prime} Y_{t}\right)$ e $C_{t}=\beta^{-}\left(\beta^{\prime} Y_{t}\right)$. Portanto, tendência e ciclo são combinações lineares de $Y_{t}$. Note que $T_{t}$ é gerado por uma combinação linear de $Y_{t}$ usando os vetores cocaracterísticos, contendo o componente de longo prazo (visto que $\tilde{\beta}^{\prime} Y_{t}$ é um componente que segue um passeio aleatório). Por outro lado, $C_{t}$ é gerado por uma combinação linear de $Y_{t}$ usando os vetores de cointegração, contendo o componente de curto prazo (porque $\beta^{\prime} Y_{t}$ é $I(0)$ e serialmente correlacionado).

\subsection{Teste de ciclos comuns}

A existência de ciclos comuns será testada a partir do procedimento de correlações canônicas delineado por Vahid e Engle (1993). O primeiro passo é estimar o VEC para descobrir os termos de correção erro da série, ou seja, para conhecer as relações de longo prazo. Retomando a equação (3):

$$
\Delta Y_{t}=\alpha \beta^{\prime} Y_{t-1}+\sum_{i=1}^{p} \Gamma_{j} \Delta Y_{t-j}+\varepsilon_{t}
$$

Definindo $\rho_{t}$ como um vetor de dimensão $(n \times 1)$ obtido como combinação linear do vetor a $\Delta Y_{t}$ e $\eta_{t}$ como o vetor de dimensão $((n p+r) x 1)$ obtido como combinação linear das defasagens de $\Delta Y_{t}$ e dos termos de correção de erro $\left(x_{t}\right)$.

$$
\begin{aligned}
& \rho_{t}=A^{\prime} \Delta Y_{t} \\
& \eta_{t}=B^{\prime}\left[\Delta Y_{t-1} \ldots \Delta Y_{t-p+1} \alpha Y_{t-1}\right]
\end{aligned}
$$


As matrizes $A_{n x n}$ e $B_{n x(n p+r)}$ são escolhidas de modo que quatro condições sejam satisfeitas. As duas primeiras afirmam que os elementos de $\rho_{t}$ e $\eta_{t}$ possuem variância unitária. A terceira condição assegura que o i-ésimo elemento de $\rho_{t}$ seja não correlacionado com o j-ésimo elemento de $\eta_{t}$, e a condição final condiciona a ordenação dos elementos de $\rho_{t}$ e $\eta_{t}$ de modo que:

$$
1 \geq \lambda_{1} \geq \ldots \geq \lambda_{n} \geq 0
$$

As correlações canônicas e os valores de $A$ e $B$ podem ser calculados a partir da matriz de covariância de $\Delta Y_{t}$ e $x_{t}$ através dos autovalores e autovetores. $O$ teste estatístico é análogo ao teste do traço do procedimento de Johansen (1988), com a hipótese nula, sendo que o espaço cocaracterístico é ao menos $s$, calculado como:

$$
C(p, s)=-(T) \sum_{i=1}^{s} \log \left(1-\lambda_{i}^{2}\right)
$$

onde $\lambda_{i}^{2}$ são as menores $s$ correlações canônicas elevadas ao quadrado. Sob a hipótese nula $C(p, s)$ segue uma distribuição $\chi^{2} \operatorname{com} s(n(P-1)+r)-s(n-s)$ graus de liberdade, sendo $n$ a dimensão do sistema ${ }^{4}$.

\subsection{Coerência e diferença de fase}

Considere um vetor de duas variáveis estacionárias $y_{t}=\left(X_{t}, Y_{t}\right)$. Seja $S_{Y Y}(w)$ o espectro populacional de $Y$, e $S_{Y X}(w)$ o espectro populacional cruzado (cross spectrum) entre $X, Y$. O cross spectrum populacional pode ser escrito em termos de seus componentes reais e imaginários como $S_{Y X}(w)=C_{Y X}(w)+i Q_{Y X}(w)$, onde $C_{Y X}(w)$ e $Q_{Y X}(w)$ denotam o cospectrum populacional e quadrature spectrum populacional entre $X, Y$, respectivamente.

A coerência (coherence populational) representa o grau de sincronia entre duas séries temporais no domínio da frequência, sendo uma medida do grau ao qual $X$ e $Y$ são conjuntamente influenciados por ciclos de frequência $w$.

$$
h_{Y X}(w)=\frac{\left[C_{Y X}(w)\right]^{2}+\left[Q_{Y X}(w)\right]^{2}}{S_{Y Y}(w) S_{X X}(w)}
$$

4 O teste de ciclos comuns foi realizado no Software Structural VAR. 
A coerência toma valores entre $0 \leq h_{Y X}(w) \leq 1$. Se $h_{Y X}(w)=1$ em algum dado ponto, então, ambas as séries caminham juntas a uma dada frequência, ou ciclo; se $h_{Y X}(w)=1$ para todo ponto espectral, assim, as séries são comuns em todas as frequências, ou ciclos.

Já o espectro cruzado, geralmente, é representado no campo dos complexos e pode ser expresso na sua forma polar:

$$
S_{Y X}(w)=C_{Y X}(w)+i Q_{Y X}(w)=R(w) \exp (i \theta(w))
$$

onde $R(w)=\left\{\left[C_{Y X}(w)\right]^{2}+\left[Q_{Y X}(w)\right]\right\}^{1 / 2}$ e $\theta(w)$ representa o ganho e o ângulo em radianos na frequência $w$. $\bigcirc$ uso da análise espectral com valores complexos tem a vantagem de computar a fase da transformação espectral de cada série proporcionando informações em relação aos atrasos das oscilações entre duas séries como função da frequência. A diferença de fase, portanto, mostra a posição relativa entre duas séries, indicando se elas se movem conjuntamente ou se há alguma relação de liderança. ${ }^{5}$

\subsection{Teste de causalidade de Granger no domínio de frequências}

Outra ferramenta espectral útil para a análise de comovimentos entre séries temporais consiste no teste de causalidade de Granger no domínio de frequências ${ }^{6}$. Farné e Montanari (2018) desenvolveram a análise de causalidade proeminente no sentido de Granger com o intervalo de confiança estabelecido por amostragem de bootstrap. A principal vantagem dessa ferramenta espectral é a avaliação de causalidade entre as séries em horizontes de curto, médio e longo prazos.

O teste de causalidade incondicional de Granger parte de um modelo $\operatorname{VAR}(k)$ bivariado, com um vetor de séries temporais $\left(X_{t}\right.$ e $\left.Y_{t}\right)$ estacionárias em covariância. $O$ espectro de $X_{t}$ (variável de efeito) com respeito à $Y_{t}$ (variável de causa) é, então, definido como em Geweke (1982):

$$
h_{Y \rightarrow X}(w)=\ln \left(\frac{h_{X X}(w)}{\tilde{P}_{X X}(w) \sigma_{X X} \tilde{P}_{X X}(w)^{*}}\right)
$$

5 As estatísticas de coerência e diferença de fase do espectro foram computadas no software $\mathrm{R}$, a partir do pacote Stats.

6 O teste de causalidade de Granger no domínio de frequências foi realizado no software R, a partir do pacote Grangers. 
onde $\tilde{P}_{X X}(w)$ é uma função de transferência, e $h_{Y \rightarrow X}(w)$ varia no intervalo $(0, \infty)$, com $w$ variando entre $(-\pi, \pi)$. Portanto, o espectro incondicional $\left(h_{Y}\right.$ $\left.\rightarrow_{X}(w)\right)$ considera todos os efeitos de valores passados de $Y_{t}$ sobre $X_{t}$ na frequência $w$. Em outras palavras, se a causalidade de Granger espectral for estatisticamente superior a zero $\left(h_{Y \rightarrow X}(w)>0\right)$, então os valores passados de $Y_{t}$ auxiliam na previsão de $X_{t}$ em ciclos de periodicidade $1 /$ w .

O teste de hipótese é baseado numa amostragem de 1.000 tiragens por bootstrap. Se a estatística de Granger espectral na frequência w for superior ao valor mediano estimado via bootstrap em um nível de significância de $5 \%$, então, rejeita-se a hipótese nula de que a série $Y$ não causa no sentido de Granger a série $X$ ao nível de frequência $w$.

Por fim, é interessante pontuar que os ciclos de referência na abordagem frequentista possuem periodicidade igual a $1 / w$, com a frequência $w$ oscilando entre (0.0 e 0.5]. Para facilitar a visualização dos ciclos de referência em interesse, aplicamos uma transformação sobre os níveis de frequência de modo que $w^{\prime}=4 w$, e os ciclos de referência passam a possuir periodicidade igual a $4 / w$. Em suma, frequências iguais a 2.0, 1.0, 0.5 e 0.25 representam ciclos com periodicidade iguais a 2, 4, 8 e 16 meses, respectivamente.

\section{Estimação empírica e base de dados}

\section{1 Base de dados}

A Pesquisa Industrial Mensal Produção Física - Brasil fornece estimativas mensais do produto real da indústria de quatorze estados do Brasil ${ }^{8}$. Como um índice conjuntural, sua importância deve-se à capacidade de indicar o comportamento efetivo da produção real da indústria com um mínimo de defasagem em relação ao período de referência (IBGE, 2004). Do ponto de vista estatístico, a alta frequência de observações torna o índice ideal para modelar a dinâmica de curto prazo da atividade industrial, em ordem de interpretar os ciclos dos negócios dos Estados observados.

7 A coleta de dados das empresas e dos respectivos produtos selecionados é realizada por unidade local de empresas selecionadas através de um questionário eletrônico ou de um formulário impresso.

8 Amazonas, Pará, Ceará, Pernambuco, Bahia, Minas Gerais, Espírito Santo, Rio de Janeiro, São Paulo, Paraná, Santa Catarina, Rio Grande do Sul, Mato Grosso e Goiás. 
Frente à limitação no número de regiões comtempladas pela pesquisa, sugere-se utilizar a decomposição das séries em componente permanente e transitório para as unidades federativas pesquisadas do Sul, Sudeste e Nordeste. A princípio a amostra incluiria os dois estados com o setor industrial mais relevante de cada região, porém, visto que São Paulo, Rio de Janeiro e Minas Gerais representam os três maiores valores agregados de atividades industriais, decidiu-se adicionar os três estados na amostra.

Os dados de produção total da indústria da Bahia, Minas Gerais, Paraná, Pernambuco, Rio de Janeiro, Rio Grande do Sul e São Paulo foram obtidos junto ao Instituto Brasileiro de Geografia e Estatística (IBGE). $O$ instituto disponibiliza as séries em forma de índice de base fixa com ajuste sazonal9 (Índice dessazonalizado, base: 2012=100). No intuito de evitar a época de forte instabilidade econômica em nível nacional, o presente estudo fez a opção de utilizar somente as observações amostrais de janeiro de 2002 até dezembro de 2014, abrangendo uma amostra de 156 observações para cada série.

Tabela 1 Descrição dos estados contemplados na pesquisa - ano 2012

\begin{tabular}{l|r|r|r}
\hline Estados & $\begin{array}{r}\text { PIB industrial - } \\
\text { em bilhões } \\
\text { de R\$ }\end{array}$ & $\begin{array}{r}\text { Participação no } \\
\text { PIB industrial } \\
\text { nacional }\end{array}$ & $\begin{array}{r}\text { Principais } \\
\text { produtos }\end{array}$ \\
\hline Bahia & 37,0 & $3,8 \%$ & $\begin{array}{r}\text { Derivados do petróleo, } \\
\text { alimentos e químicos }\end{array}$ \\
\hline Paraná & 53,2 & $5,5 \%$ & $\begin{array}{r}\text { Alimentos, derivados do } \\
\text { petróleo e veículos }\end{array}$ \\
\hline Pernambuco & 24,9 & $2,6 \%$ & $\begin{array}{r}\text { Alimentos, químicos e extração } \\
\text { de petróleo e gás natural }\end{array}$ \\
\hline Minas Gerais & 103,4 & $10,7 \%$ & $\begin{array}{r}\text { Extrações de minerais e metálicos, } \\
\text { alimentos e metalurgia }\end{array}$ \\
\hline Rio de Janeiro & 138,1 & $14,3 \%$ & $\begin{array}{r}\text { Extração do petróleo e gás natural, } \\
\text { derivados do petróleo e metalurgia }\end{array}$ \\
\hline Rio Grande do Sul & 60,1 & $6,2 \%$ & $\begin{array}{r}\text { Alimentos, veículos, } \\
\text { máquinas e equipamentos }\end{array}$ \\
\hline São Paulo & 288,6 & $29,8 \%$ & $\begin{array}{r}\text { Alimentos, derivados do } \\
\text { petróleo e químicos }\end{array}$ \\
\hline
\end{tabular}

Fonte: $\mathrm{CNI}$.

9 O ajuste sazonal das séries foi obtido utilizando-se o software X-12 ARIMA, U.S. Census Bureau. 
Segundo a Tabela 1, observa-se que o perfil de produção regional não se dá de forma homogênea. A produção de alimentos desponta como a principal atividade para o estado do Paraná, Pernambuco, Rio Grande do Sul e São Paulo. Enquanto o Rio de Janeiro e a Bahia possuem a extração e fabricação de derivados do petróleo como principal atividade, respectivamente.

Diferenças na estrutura regional industrial podem contribuir para diferenças no comportamento dos ciclos econômicos. Uma vez que as regiões possuem diferentes composições de indústria elas experimentam diferentes choques de produto, resultando em específicos ciclos econômicos. Por outro lado, a integração entre as regiões e o maior comércio pode surgir como indutora da propagação dos ciclos entre os estados de diferentes regiões. A existência de um comportamento similar entre a dinâmica de resposta dos estados a esses choques evidencia a presença de comovimento entre eles.

\section{Figura 1 Trajetória temporal do produto industrial mensal}

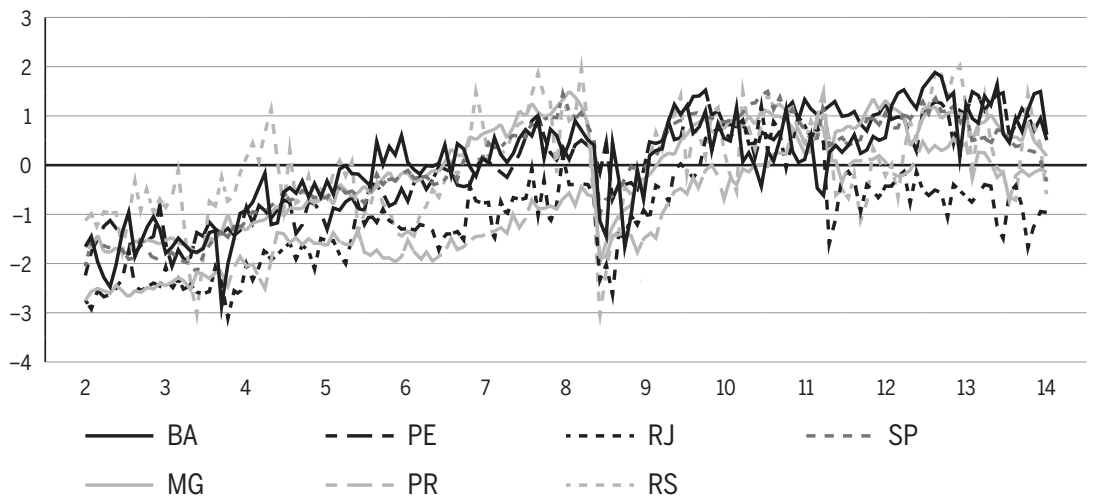

Fonte: Elaborada pelos autores.

Nota: Os dados foram normalizados.

A Figura 1 representa a trajetória temporal da produção industrial dos estados durante o período estudado. Analisando a trajetória comum dos estados é possível notar movimentos oscilatórios em torno da média na produção industrial entre 2002 até o final de 2003. Após isso, a produção se tornou crescente até o último trimestre de 2008, quando houve uma grande depressão na taxa de produção de todos os estados, com destaque para o Rio Grande do Sul e Minas Gerais. Essa forte queda é reflexo da crise internacional instaurada a partir de setembro de 2008 e da política 
monetária para contenção da inflação, como a elevação da taxa de juros. Posteriormente, houve um pequeno período de recuperação da economia até meados de 2010, desde então até o fim da amostra verifica-se um período de alta volatilidade.

Individualmente, cabe destacar que Paraná e Rio de Janeiro sofreram outro período de forte queda na tendência do produto industrial no final de 2011 e começo de 2012.

Portanto, há momentos de mudança repentina no comportamento das séries indicando que pode haver quebras estruturais na série. Visto isso, uma dummy será adicionada para captar o efeito dessa quebra nos testes de raiz unitária e de cointegração, tornando os resultados robustos à mudança estrutural.

\subsection{Testes de estacionariedade}

Uma das condições necessárias para que a decomposição em tendências e ciclos sugerida por Vahid e Engle (1993) seja válida é que todas as séries sejam integradas de ordem $1-\mathrm{I}(1)$.

Tabela 2 Testes de estacionariedade incorporando quebra estrutural

\begin{tabular}{|c|c|c|c|c|c|c|c|c|}
\hline & & BA & MG & PE & PR & RJ & RS & SP \\
\hline $\begin{array}{l}\text { Zivot-Andrews (1992) } \\
\text { (H0: Série com R.U.) }\end{array}$ & Teste em nível & $-4,65$ & $-4,83$ & $-6,57$ & $-4,98^{*}$ & $-4,62$ & $-5,17^{* *}$ & $-2,89$ \\
\hline $\begin{array}{l}\text { Unit Root with } \\
\text { Break Test (1996) } \\
\text { (H0: Série com R.U.) }\end{array}$ & Teste em nível & $-3,79$ & $-3,77$ & $-3,63$ & $-5,06^{* *}$ & $-5,23 * *$ & $-5,34^{* *}$ & $-2,77$ \\
\hline $\begin{array}{l}\text { Perron (1997) } \\
\text { (HO: Série com R.U.) }\end{array}$ & Teste em nível & $-4,66$ & $-4,77$ & $-6,58^{* * *}$ & $-5,58^{*}$ & $-5,94^{* *}$ & $-5,34^{*}$ & $-3,61$ \\
\hline $\begin{array}{l}\text { Lanne et al. (2002) } \\
\text { (H0: Série com R.U.) }\end{array}$ & Teste em nível & $-1,68$ & $-1,37$ & $-1,24$ & $-0,77$ & $-1,40$ & $-1,67$ & $-1,48$ \\
\hline
\end{tabular}

Fonte: Elaborado pelos autores.

Todos os testes foram feitos levando em conta a presença de um intercepto e uma tendência.

Estatística treportada. Escolha de defasagens, segundo o critério de Schwarz, com limitação máxima de 12 defasagens * Rejeição da hipótese nula a 10\%, ${ }^{*}$ Rejeição da hipótese nula a 5\%, ${ }^{* *}$ Rejeição da hipótese nula a $1 \%$.

A Tabela 2 reporta que todas as séries - à exceção de Paraná, Rio de Janeiro e Rio Grande do Sul a um nível de 5\%, segundo o teste Unit Root with Break 
Test, Rio Grande do Sul segundo o teste de Zivot-Andrews e Pernambuco segundo o teste de Perron - apresentam-se não estacionárias em nível, mas estacionárias em $1^{a}$ diferença. ${ }^{10}$ Logo, os resultados indicam que a taxa de produção industrial para as sete unidades federativas estudadas seguem um processo integrado de primeira ordem. ${ }^{11}$

\subsection{Teste de cointegração}

Visando lidar com a presença de quebra estrutural, foi implementado o teste de Chow, cujo objetivo é testar a igualdade de um conjunto de coeficientes entre duas regressões a partir da estatística F, para testar a hipótese nula de quebra estrutural no período de novembro de 2008 - alguns meses após o ápice da crise mundial. A estatística de Chow não rejeita a hipótese nula de mudança estrutural no período de novembro de 2008 para todas as variáveis estudadas, havendo instabilidade no modelo no período anterior a essa data e estabilidade logo após.

A partir daí é possível estimar as relações de equilíbrio de longo prazo entre as variáveis através do teste de cointegração de Johansen et al. (2000), o qual incorpora uma dummy associada à quebra estrutural identificada em novembro de 2008 na determinação de seus valores críticos.

Tabela 3 Teste de cointegração de Johansen et al. (2000)

\begin{tabular}{|c|c|c|c|}
\hline \multicolumn{4}{|l|}{ Teste do traço } \\
\hline $\mathrm{N}^{0}$ de vetores & Estatística do traço & Valores críticos a $5 \%$ & p-valor \\
\hline$r=0$ & 286,70 & 158,31 & 0,0000 \\
\hline$r \leq 1$ & 208,64 & 124,51 & 0,0000 \\
\hline$r \leq 2$ & 152,82 & 94,63 & 0,0000 \\
\hline$r \leq 3$ & 104,55 & 68,66 & 0,0000 \\
\hline$r \leq 4$ & 62,17 & 46,63 & 0,0001 \\
\hline$r \leq 5$ & 27,00 & 28,41 & 0,0738 \\
\hline$r \leq 6$ & 4,06 & 14,00 & 0,8181 \\
\hline
\end{tabular}

Fonte: Elaborada pelos autores.

10 Resultados disponíveis no Apêndice.

11 O decaimento lento observado na Função Autocorrelação das séries temporais e o sinal mais intenso observado em baixas frequências no periodograma dão robustez à hipótese de não estacionariedade das séries de produção industrial dos estados. 
Utiliza-se uma defasagem de acordo com critério de Schwarz, considerando-se a presença de intercepto e tendência.

O resultado do teste do traço de Johansen et al. (2000) aponta a presença de cinco vetores cointegrantes a um nível de $5 \%$ de significância. Consequentemente, existem duas tendências comuns entre as séries - visto que o número de tendências comuns é dado pela diferença entre o número de variáveis $(n=7)$ e o espaço de cointegração $(r=5)$ e, no máximo, cinco ciclos comuns.

Assim, a dinâmica temporal do índice de produção industrial das séries analisadas é determinada por dois componentes agregados comuns e por características individuais presentes em cada estado.

$\bigcirc$ procedimento de Johansen et al. (2000) permite, também, normalizar o vetor de cointegração possibilitando, assim, identificar as relações econômicas das séries em termos de elasticidade. A normalização foi feita de forma que as $r$ primeiras séries no vetor $x_{t}$ formam uma matriz identidade.

Tabela 4 Vetor de cointegração normalizado

\begin{tabular}{rr|r|r|r|r|r}
\hline \multicolumn{1}{l}{ Vetor de cointegração $\left(\boldsymbol{\alpha}_{\text {rxn }}\right)$} & & & & & \\
\hline BA & MG & PE & PR & RJ & RS & SP \\
\hline 1,00 & 0,00 & 0,00 & 0,00 & 0,00 & $-0,093$ & $-0,734$ \\
\hline 0,00 & 1,00 & 0,00 & 0,00 & 0,00 & $-0,151$ & $-0,941$ \\
\hline 0,00 & 0,00 & 1,00 & 0,00 & 0,00 & $-0,137$ & $-0,607$ \\
\hline 0,00 & 0,00 & 0,00 & 1,00 & 0,00 & $-0,741$ & $-0,461$ \\
\hline 0,00 & 0,00 & 0,00 & 0,00 & 1,00 & 0,192 & $-0,637$ \\
\hline
\end{tabular}

Vetor de cointegração aplicado às séries mensais acumuladas à taxa anual para as regiões metropolitanas do Brasil durante o período de outubro de 1995 até dezembro de 2014. O VEC foi estimado levando em conta a presença de intercepto e tendência e utilizando a defasagem de acordo com o critério de Schwarz.

Em termos econômicos, os resultados mostram que um choque permanente positivo de $1 \%$ na taxa de atividade industrial mensal em São Paulo causa uma variação permanente e positiva de $0,734 \%$ no nível de produção do estado da Bahia, por exemplo. Avaliando o efeito de um choque permanente e positivo na atividade industrial de São Paulo e Rio Grande do Sul sobre a atividade econômica dos demais estados, nota-se que o primeiro causa um maior impacto no comportamento da atividade industrial de todos estados, à exceção do Paraná. Esse resultado está associado às características regionais, uma vez que os choques permanentes na atividade do Rio 
Grande do Sul causam um menor impacto nas regiões sudeste e nordeste, demonstrando maior interação intrarregional de longo prazo na região Sul. Adicionalmente, pode-se notar que desvios permanentes e positivos sobre atividade paulista causam maior efeito sobre o estado de Minas Gerais $(0,941 \%)$ e um menor efeito sobre o estado do Paraná (0,461\%). Tais resultados indicam uma relação inelástica entre o comportamento de longo prazo da taxa de atividade industrial das regiões Sul e Sudeste.

\subsection{Teste de ciclos comuns}

Para examinar a existência de dinâmicas de curto prazo comum entre as séries procedeu-se ao teste de correlações canônicas. $O$ método testa a hipótese nula de que a correlação canônica corrente e todas as correlações canônicas menores são estatisticamente iguais a zero. Como observado anteriormente, a rank cocaracterístico $s$ é igual ao número de correlações canônicas estatisticamente iguais a zero.

Foi verificado que a hipótese da existência de dois vetores de cocaracterização não pode ser rejeitada, a um nível de significância de 10\% e, consequentemente, é confirmada a presença de cinco ciclos comuns na amostra.

Tabela 5 Teste de correlações canônicas

\begin{tabular}{l|rrrrr}
\hline Teste do traço & \multicolumn{1}{c}{$\begin{array}{l}\text { Correlação } \\
\text { canônica2 }\end{array}$} & $\mathbf{C ( p , s )}$ & $\begin{array}{r}\text { Graus de } \\
\text { liberdade }\end{array}$ & p-valor \\
\hline$s>0$ & 0,109 & 7,899 & 5 & 0,162 \\
\hline$s>1$ & 0,143 & 18,350 & 12 & 0,105 \\
\hline$s>2$ & 0,175 & 31,383 & 21 & 0,067 \\
\hline$s>3$ & 0,305 & 56,067 & 32 & 0,005 \\
\hline
\end{tabular}

Fonte: Elaboração do autor.

A ordem de defasagem selecionada para estimação do VEC e, por consequência, do teste de ciclos comuns segue o critério de Akaike. A escolha desse critério se deu porque o teste só pode ser realizado num VEC de ordem igual ou superior a dois.

A existência de dois vetores de cocaracterização e cinco vetores de cointegração entre as séries implica que a matriz $\mathrm{A}=\left[\tilde{\alpha}^{\prime} \alpha^{\prime}\right]$ possui posto pleno, logo é possível obter as tendências e ciclos individuais através da partição 
da matriz $A$, assim como encontrar os ciclos comuns pela combinação dos vetores de cointegração e das variáveis do sistema $\left(\alpha^{\prime} Y_{t}=\alpha^{\prime} C_{t}\right)$.

\subsection{Ciclos comuns: análise no domínio do tempo}

Realizado o teste de ciclos comuns, a próxima etapa do presente trabalho será extrair os cinco ciclos comuns $(n-s=7-2=5)$ a partir de $\alpha^{\prime} Y_{t}=\alpha^{\prime} C_{t}$, e os componentes de ciclos individuais de cada região metropolitana através da equação $\alpha^{-}\left(\alpha^{\prime} Y_{t}\right)$ nas Figuras 4, 5 e 6. Cabe fazer uma ressalva que, devido à delimitação de espaço, somente os ciclos comuns \#2 e \#4 serão representados aqui pelo fato de ambos terem sido os únicos a possuírem valores relevantes de correlação com os ciclos individuais.

Figura 2 Ciclos comuns e individuais

\section{2a Ciclos comuns}

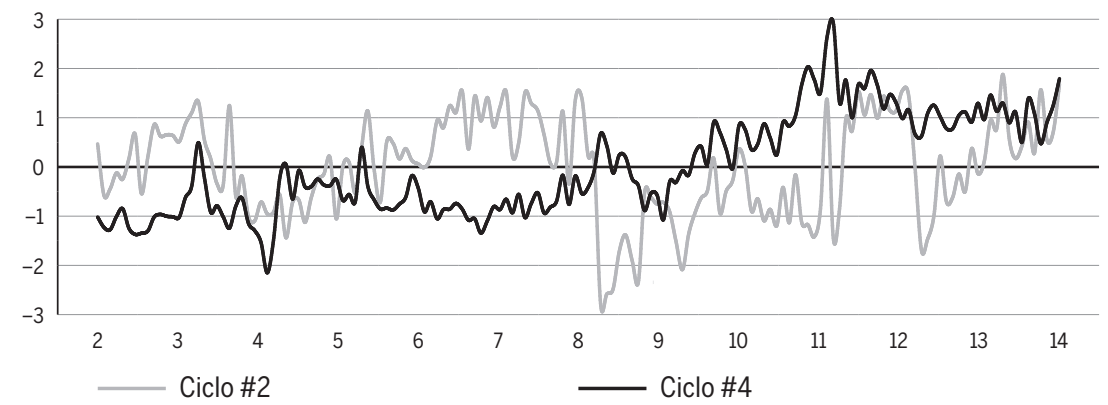

\section{2b Ciclos da região Nordeste}

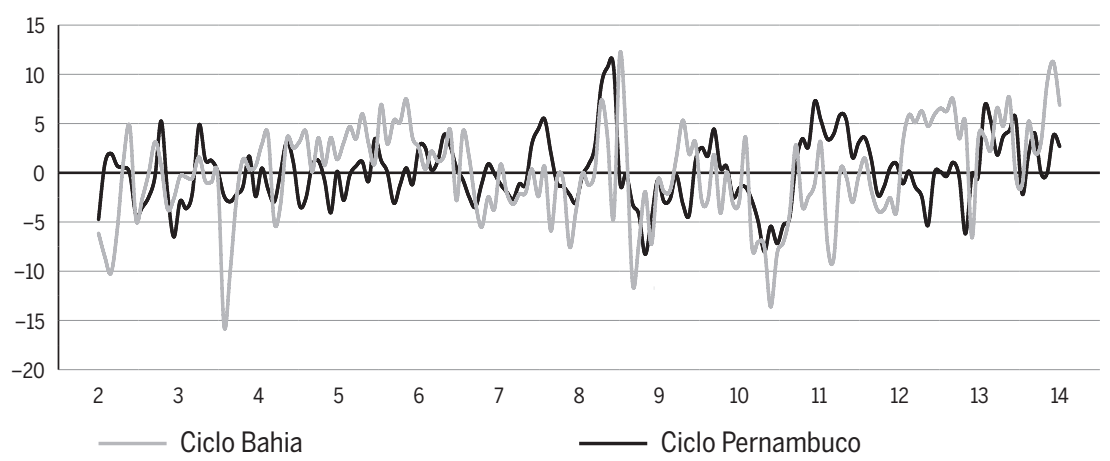




\section{2c Ciclos da região Sul}

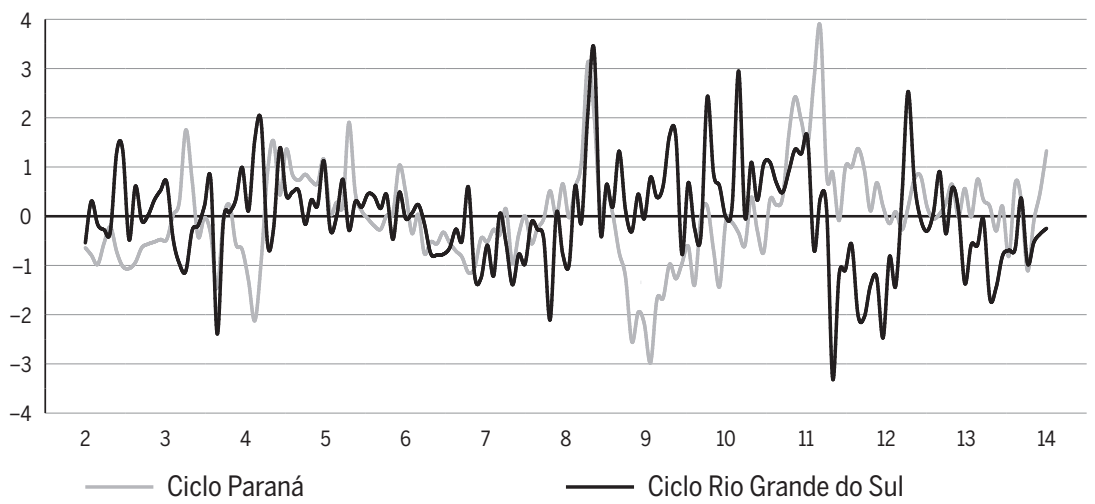

2d Ciclos da região Sudeste

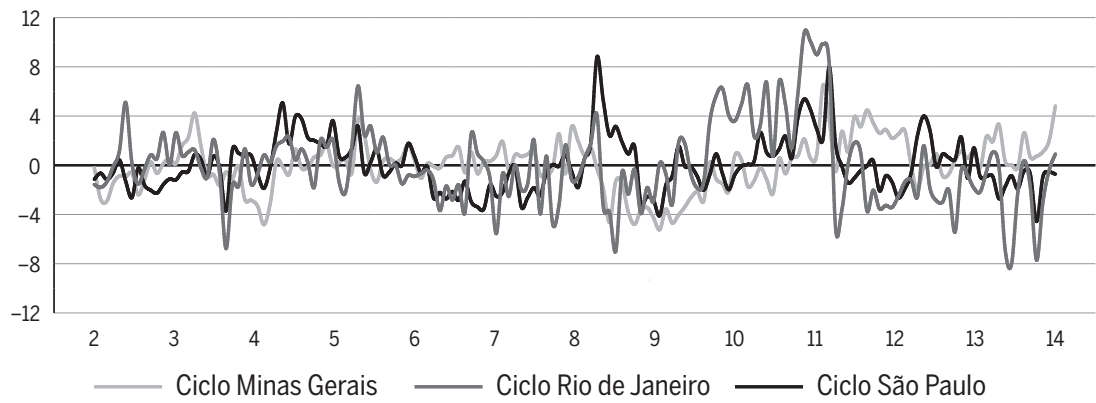

Fonte: Elaborada pelos autores.

Nota: Os ciclos comuns (2a) foram normalizados para representação gráfica.

O ciclo comum \#2 aparenta possuir volatilidade menor, se comparado com o ciclo comum \#4. Consoante à esfera regional, nota-se um padrão próximo entre os ciclos da Bahia e Pernambuco, com o primeiro apresentando maiores valores extremos negativos. Na região Sul, nota-se que após o advento da crise mundial, os ciclos do Rio Grande do Sul e Paraná passaram a possuir comportamento assimétrico. Por fim, os ciclos de negócios de Minas Gerais apresentaram comportamento menos volátil do que os de São Paulo e Rio de Janeiro.

O ciclo comum \#2 apresenta valores de mínimo, máximo e desvio padrão mais próximos aos ciclos individuais, enquanto o ciclo comum \#4 é robustamente mais volátil. Analisando os valores extremos destaca-se 
o estado do Paraná, com o maior valor máximo da amostra $(22,545)$ e o valor mínimo mais acentuado $(-17,472)$, bem como o maior nível de volatilidade $(5,867)$. Os ciclos individuais possuem coeficientes de assimetrias em direções distintas, contudo em baixas magnitudes. Todos os ciclos apresentaram leptocurtose - possuindo caudas pesadas e distribuição de probabilidade mais achatada que a normal - com destaque para os estados do Paraná e São Paulo.

Tabela 6 Estatística descritiva dos ciclos - 2012:01 a 2014:12

\begin{tabular}{lrrrr|r|r|r|r|r|r}
\hline Métrica & $\begin{array}{r}\text { Ciclo } \\
\mathbf{\# 2}\end{array}$ & $\begin{array}{r}\text { Ciclo } \\
\mathbf{\# 4}\end{array}$ & $\mathbf{B A}$ & $\mathbf{M G}$ & $\mathbf{P E}$ & $\mathbf{P R}$ & $\mathbf{R J}$ & $\mathbf{R S}$ & $\mathbf{S P}$ \\
\hline Média & 0,000 & 0,000 & 0,000 & 0,000 & 0,000 & 0,000 & 0,000 & 0,000 & 8,784 \\
\hline Máximo & 5,733 & 26,31 & 12,084 & 6,506 & 10,716 & 22,545 & 10,759 & 3,975 & 8,784 \\
\hline Mínimo & $-8,472$ & $-19,353$ & $-15,586$ & $-5,236$ & $-8,292$ & $-17,472$ & $-8,053$ & $-3,889$ & $-4,571$ \\
\hline D. padrão & 3,049 & 8,997 & 4,782 & 2,082 & 3,342 & 5,867 & 3,344 & 1,176 & 2,187 \\
\hline Assimetria & $-0,327$ & 0,483 & $-0,402$ & $-0,093$ & 0,381 & 0,469 & 0,643 & 0,146 & 0,963 \\
\hline Curtose & 2,605 & 2,372 & 3,2929 & 3,397 & 3,691 & 4,830 & 4,182 & 4,360 & 4,867 \\
\hline
\end{tabular}

Fonte: Elaborada pelos autores.

Os ciclos comuns foram subtraídos pelos seus respectivos interceptos.

É possível visualizar maiores detalhes desses componentes cíclicos ao utilizar a análise de correlação cruzada de forma a avaliar a relação mútua entre as variáveis.

Tabela 7 Correlação entre os ciclos individuais e comuns - 2012:01 a 2014:12

\begin{tabular}{lr|r|r|r|r|r|r|r|r|r}
\hline Ciclos & $\begin{array}{r}\text { Ciclo } \\
\text { \#2 }\end{array}$ & $\begin{array}{r}\text { Ciclo } \\
\text { \#4 }\end{array}$ & BA & MG & PE & PR & RJ & RS & SP \\
\hline Ciclo \#2 & 1,000 & & & & & & \\
\hline Ciclo \#4 & $-0,003$ & 1,000 & & & & & & & \\
\hline BA & 0,045 & 0,134 & 1,000 & & & & & & \\
\hline MG & 0,681 & 0,525 & 0,186 & 1,000 & & & & & \\
\hline PE & 0,029 & 0,313 & 0,272 & 0,222 & 1,000 & & & & \\
\hline PR & $-0,045$ & 0,627 & 0,193 & 0,677 & 0,447 & 1,000 & & & \\
\hline RJ & $-0,210$ & 0,201 & $-0,178$ & 0,144 & $-0,005$ & 0,363 & 1,000 & & \\
\hline RS & $-0,701$ & $-0,132$ & 0,013 & $-0,497$ & $-0,121$ & 0,014 & 0,435 & 1,000 & \\
\hline SP & $-0,666$ & 0,384 & 0,118 & 0,071 & 0,183 & 0,751 & 0,421 & 0,433 & 1,000 \\
\hline
\end{tabular}

Fonte: Elaborada pelos autores. 
De acordo com a Tabela 7 o ciclo comum \#2 se relaciona de forma mais robusta com o ciclo do Rio Grande do Sul (associação linear negativa) e com os ciclos individuais da região Sudeste, exceto Rio de Janeiro - sendo correlacionado de forma positiva com o ciclo de Minas Gerais e negativa com São Paulo -, enquanto o ciclo comum \#4 se relaciona de forma positiva com os ciclos individuais de Minas Gerais e Paraná. Tal comportamento indica que os movimentos transitórios causados pelos ciclos comuns nos ciclos individuais são direcionados pelos ciclos das regiões Sul e Sudeste, com a região Nordeste possuindo baixo nível de correlação com os ciclos comuns - oscilando entre 0,045 e 0,313 . Nesse sentido, o resultado reforça que a evolução do setor industrial nacional está fortemente relacionada com a dinâmica das regiões Sul e Sudeste, às quais possuem maior propensão ao desenvolvimento de indústrias com maior intensidade tecnológicas, conforme observado em Monteiro e Lima (2017).

Sobre os ciclos individuais nota-se que o ciclo do Paraná é o que apresenta níveis de correlação mais altos com os demais estados, com destaque para a correlação com o ciclo de São Paulo - 0,751 - e de Minas Gerais 0,677. Esse resultado ressalta a importância de avaliar os componentes de curto prazo de forma separada dos componentes de longo prazo, uma vez que, apesar do alto nível de correlação de longo prazo entre Paraná e Rio Grande do Sul, no curto prazo os ciclos dos estados são independentes.

Tabela 8 Causalidade de Granger entre os ciclos individuais.

\begin{tabular}{l|rrrr|r|r|r|r}
\hline $\begin{array}{l}\text { Causali- } \\
\text { dade }\end{array}$ & BA & MG & PE & PR & RJ & RS & SP \\
\hline BA & & 0,959 & $0,046^{*}$ & 0,868 & $0,0427^{*}$ & $0,010^{*}$ & 0,882 \\
\hline MG & 0,850 & & $0,016^{*}$ & 0,324 & $0,0276^{*}$ & $0,043^{*}$ & 0,197 \\
\hline PE & 0,302 & 0,545 & & 0,811 & 0,202 & 0,481 & 0,980 \\
\hline PR & 0,999 & 0,640 & $0,005^{*}$ & & 0,187 & $0,034^{*}$ & 0,204 \\
\hline RJ & 0,480 & 0,980 & 0,084 & 0,660 & & $0,000^{*}$ & 0,392 \\
\hline RS & 0,403 & 0,202 & 0,558 & 0,083 & 0,733 & & 0,074 \\
\hline SP & 0,546 & 0,771 & 0,358 & 0,205 & 0,826 & $0,001^{*}$ & \\
\hline
\end{tabular}

Fonte: Elaborada pelos autores.

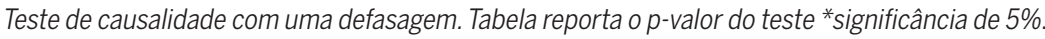

A análise do teste de causalidade entre os ciclos individuais sugere que o ciclo da Bahia e Minas Gerais possui uma capacidade preditiva quanto 
aos ciclos de Pernambuco, Rio de Janeiro e Rio Grande do Sul, não sendo previstos por nenhum dos três estados. Os choques do Rio Grande do Sul são previstos por todos os ciclos individuais, à exceção do ciclo de Pernambuco. Destaca-se também que, assim como o ciclo da Bahia e Minas Gerais, os ciclos do Paraná e São Paulo não são previstos por nenhum dos ciclos individuais.

Tabela 9 Causalidade de Granger entre os ciclos individuais e comuns.

\begin{tabular}{l|r|r|r|r|r|r|r}
\hline Causalidade & BA & MG & PE & PR & RJ & RS & SP \\
\hline $\begin{array}{l}\text { C. Comum 2 > } \\
\text { C. Individual }\end{array}$ & 0,776 & 0,667 & 0,582 & 0,327 & 0,244 & $0,035^{*}$ & 0,160 \\
\hline $\begin{array}{l}\text { C. Individual > } \\
\text { C. Comum 2 }\end{array}$ & 0,906 & 0,230 & 0,528 & 0,327 & 0,244 & $0,019^{*}$ & 0,220 \\
\hline $\begin{array}{l}\text { C. Comum 4 > } \\
\text { C. Individual }\end{array}$ & 0,997 & 0,312 & $0,021^{*}$ & 0,932 & 0,498 & 0,078 & 0,159 \\
\hline $\begin{array}{l}\text { C. Individual > } \\
\text { C. Comum 4 }\end{array}$ & 0,978 & 0,146 & 0.383 & 0,090 & 0,740 & 0,244 & 0,465 \\
\hline
\end{tabular}

Fonte: Elaborada pelos autores.

Teste de causalidade com um lag defasagem. Tabela reporta o $p$-valor do teste * significância de $5 \%$.

Conforme a Tabela 9, nenhum dos ciclos individuais foi capaz de prever o ciclo comum \#2, bem como o ciclo comum \#4 não é capaz de prever nenhum dos ciclos individuais, a exceção da causalidade de ambos os lados entre o ciclo do Rio Grande do Sul e o ciclo comum \#2. Já em relação ao ciclo comum \#4, observa-se que o ciclo individual do Paraná aparece como o único capaz de prevê-lo, enquanto o mesmo possui capacidade preditiva sobre o ciclo de negócios de Pernambuco.

\subsection{Ciclos comuns: análise no domínio da frequência}

Os resultados obtidos no subcapítulo anterior sugerem uma possível sincronização entre o ciclo do Paraná e os ciclos de Minas Gerais e São Paulo, dada a existência do forte nível de correlação temporal e a inexistência de capacidade preditiva entre os pares. No intuito de dar robustez à hipótese de comovimentos entre o ciclo paranaense e os ciclos da região Sudeste, 
será feita uma análise de sincronia no domínio da frequência através dos conceitos de coerência e diferença de fase.

A coerência refere-se ao grau de influência em conjunto dos pares de ciclos num dado nível de frequência w; assim como o conceito de correlação temporal, a coerência varia em um intervalo entre 0 e 1 . Duas séries são ditas perfeitamente correlacionadas no domínio da frequência se sua coerência é igual a 1. Já a diferença de fase é uma medida de espectro de fase entre dois ciclos na frequência $w$; se dois ciclos oscilam conjuntamente numa dada frequência $w$, diz-se que sua diferença de fase é igual a zero.

Figura 3 Coerência e diferença de fase do espectro

\section{Coerência entre os Estados}

PR \& SP

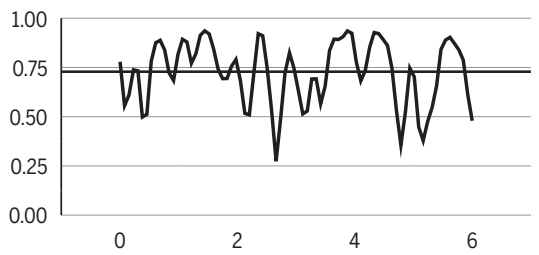

$P R \& M G$

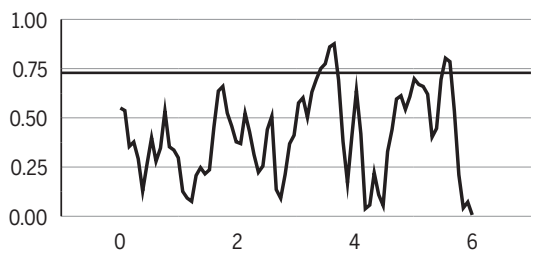

\section{PR \& RJ}

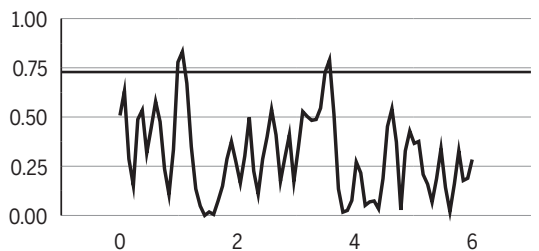

\section{Diferença de Fase entre os Estados}

PR \& SP

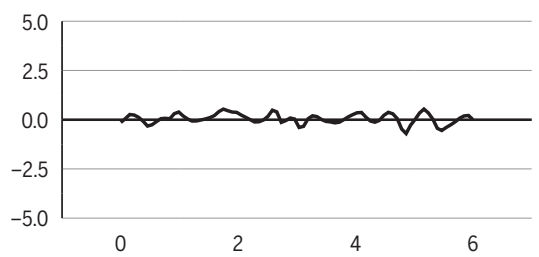

PR \& MG

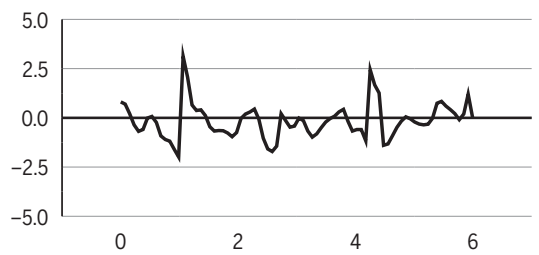

\section{PR \& RJ}

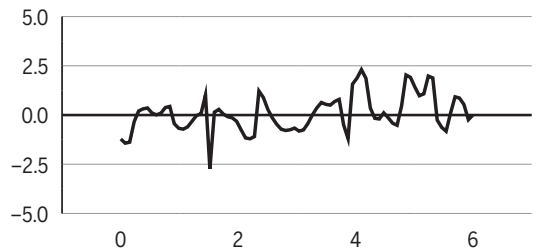

Fonte: Elaborada pelos autores. 
Logo, aliados as métricas estáticas temporais os conceitos de coerência e diferença de fase disponibilizam informações adicionais acerca do grau de sincronização entre os ciclos de negócios. Para haver sincronização, a correlação e a coerência devem ser próximas de um, e a diferença de fase deve oscilar em torno de zero ao longo de diferentes frequências.

$\mathrm{Na}$ Figura 3, os gráficos à esquerda representam os resultados para a coerência, enquanto o gráfico a direita diz respeito à diferença de fase do espectro. Já os resultados do teste de causalidade de Granger espectral estão reportados na Figura 4. Neste caso a linha horizontal reflete o coeficiente mínimo de significância estatística, a partir do qual rejeita-se a hipótese nula de que a série $Y$ não causa, no sentido de Granger, a série $Y$ na frequência $w$. Por fim, é importante relembrar que as frequências estão normalizadas, de modo que se a frequência é w então o ciclo possui duração de 4/w períodos, ou meses nesse caso. Logo, $w=0,5$ indica um ciclo de 8 meses de duração.

Figura 4 Teste de causalidade de Granger no domínio da frequência

PR causa granger SP

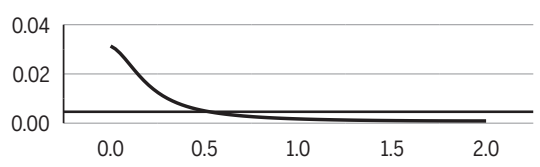

\section{PR causa granger MG}

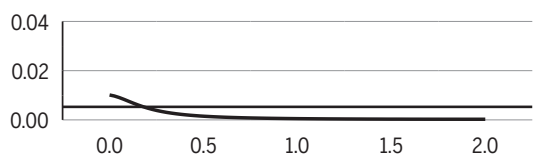

\section{PR causa granger $R J$}

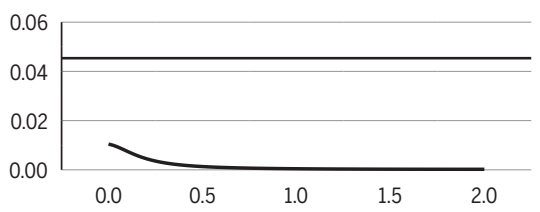

SP causa granger PR

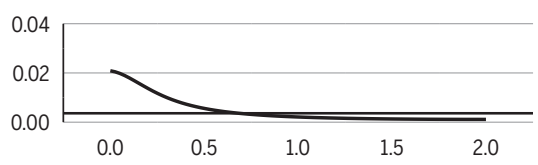

MG causa granger PR

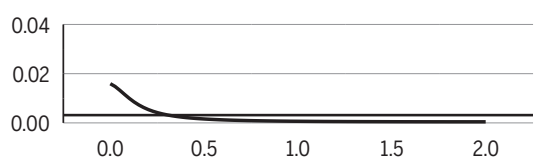

\section{$\mathrm{RJ}$ causa granger $\mathrm{PR}$}

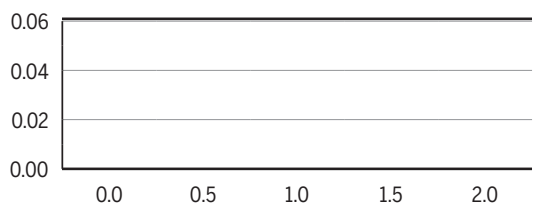

Fonte: Elaborada pelos autores. 
No caso Paraná-São Paulo a coerência encontra-se acima de 0,75 durante grande parte do intervalo de frequências, e a diferença de fase não é estatisticamente diferente de zero em nenhum intervalo frequencial. ${ }^{12} \mathrm{O}$ teste de causalidade de Granger no domínio de frequências indica bicausalidade no horizonte de médio e longo prazos, e inexistência de padrão de liderança nas oscilações nas frequências de curto prazo. Assim, os resultados encontrados a partir de ferramentas no domínio da frequência corroboram os resultados encontrados no domínio temporal. Logo, o grau de associação entre os ciclos de negócio entre Paraná e São Paulo foi confirmado através da análise espectral.

No que se refere à associação entre Paraná e Minas Gerais, o grau de coerência encontrado aponta para a rejeição de hipótese de comovimentos entre os estados, na medida em que os coeficientees de coerência não são significantes em quase todos os pontos. Os estados, porém, apresentaram diferenças de fase próximas de zero. Assim como no caso anterior, o teste de causalidade de Granger espectral rejeitou a hipótese de inexistência de causalidade no horizonte de médio e longo prazos ( $w<0,25$, intervalo que contém ciclos com durações iguais ou superiores a 16 meses) em ambas as direções, e não rejeitou ausência de causalidade no horizonte de curto prazo.

Por fim, o resultado da coerência e diferença de fase entre os ciclos de negócios do Paraná e Rio de Janeiro confirmaram a ausência de associação linear entre os estados, com baixo nível de correlação linear em nível de frequência e padrões assimétricos de diferença de fase ao longo das frequências. $O$ Rio de Janeiro apresentando comportamento defasado para ciclos de médio e longo prazos $(w<0,25)$ e precedência temporal para ciclos de curtíssimo prazo $(1,5<w<0,75)$, anulando o resultado anterior e confirmando o resultado do teste de causalidade Granger no domínio do tempo e da frequência para ciclos de curto, médio e longo prazos, indicando ausência de causalidade entre ambos os estados.

\section{Considerações finais}

O objetivo deste artigo foi caracterizar os relacionamentos dos ciclos de negócios entre atividade industrial da Bahia, Minas Gerais, Pernambuco, 12 Os resultados que mostram os intervalos de confiança podem ser solicitados diretamente aos autores. 
Paraná, Rio de Janeiro, Rio Grande do Sul e São Paulo no período de janeiro de 2002 a dezembro de 2014; ou seja, avaliar o grau de sincronização das dinâmicas de curto prazo dos principais estados do Brasil em termos de produção industrial.

Devido à presença de quebra estrutural nas séries temporais, foram realizados testes de raiz unitária com e sem incorporação de mudança estrutural. Os resultados, em alguns casos, reportaram inversões no resultado do teste de raiz unitária com e sem incorporação da quebra, segurindo que as séries podem seguir um processo integrado de ordem fracionária, devido à presença de choques de longa persistência. Sugere-se, como pesquisa futura, um avanço na discussão sobre a natureza da ordem de integração das séries de produção industrial, estendendo a modelagem da dependência temporal do caso dicotômico, I(0) ou I(1), para uma estrutura mais flexível, como a observada em Mayoral et al. (2011) ${ }^{13}$.

A análise mostrou que, mesmo na presença de uma quebra estrutural em novembro de 2008, as séries temporais dos estados são cointegradas, compartilhando de duas relações de equilíbrio de longo prazo. Os sinais dos coeficientes das elasticidades de longo prazo das séries foram consistentes, demonstrando que desvios permanentes e positivos no nível de produção industrial de São Paulo afetam de forma mais veemente as atividades industriais dos outros estados da amostra, se comparados com choques permanentes no nível de produção industrial do Rio Grande do Sul. A única exceção foi para o caso do Paraná, onde o ciclo demonstrou maior elasticidade perante choques de longo prazo oriundos do Rio Grande do Sul, o que denota maior interação intrarregional de longo prazo na região Sul. Resultado corroborado pelo fato de o ciclo do Paraná ser o menos afetado por choques da atividade industrial de São Paulo.

Assim, em termos de estratégia e condução de política econômica, o fato de as regiões compartilharem de tendências estocásticas comuns sugere que as políticas fiscais implantadas exercem efeitos permanentes nas taxas de produção industrial de todas as regiões estudadas.

Em termos de dinâmica de curto prazo, viu-se que as regiões da amostra compartilham de cinco ciclos comuns. A análise de correlação apontou, ainda, que existe uma associação linear positiva entre os ciclos do estado do Paraná e ciclos de negócios de Minas Gerais e São Paulo, com valores de 
0,655 e 0,751, respectivamente. Consoante à dinâmica de curto prazo dos demais estados, observou-se certo grau de independência entre os ciclos, uma vez que as demais correlações não ultrapassaram o patamar de 0,5.

Em relação à dinâmica dos ciclos individuais, o teste de causalidade apurou que os ciclos da Bahia e Minas Gerais causam, no sentido de Granger, os ciclos de Pernambuco, Rio de Janeiro e Rio Grande do Sul a um nível de significância de 5\%. Verificou-se também que nenhum dos ciclos individuais foi capaz de prever o ciclo comum \#2, à exceção do Rio Grande do Sul, que fora também causado no sentido de Granger pelo ciclo comum \#2. Já o ciclo individual do estado do Paraná apresentou precedência temporal em relação à dinâmica do ciclo comum \#4.

Dessa forma, a ausência de capacidade preditiva entre os pares de ciclos econômicos do estado de Paraná-Minas Gerais e Paraná-São Paulo corroboraram a hipótese de associação linear entre o ciclo desses estados. Para verificar a hipótese de sincronização entre os pares de ciclos foi realizada a análise de comovimentos no domínio da frequência, através de ferramentas de análise espectral.

Segundo os resultados no domínio da frequência, foi confirmada a hipótese de sincronização entre os ciclos do Paraná e São Paulo, enquanto a hipótese de comovimentos entre o ciclo do Paraná e Minas Gerais foi rejeitada.

Os resultados encontrados contribuem para a literatura de ciclos de negócios regionais na medida em que lança mão de ferramentas adicionais para a análise de sincronização. Pode-se verificar que o grau de associação linear de longo prazo não foi mantido no curto prazo - como a simultânea correlação linear forte de longo prazo entre Paraná e Rio Grande do Sul e a independência de seus ciclos, por exemplo - acentuando a importância da diferenciação dos impactos permanentes e transitórios sobre a economia.

\section{Referências}

BANERJEE, A.; LUMSDAINE, R. L; STOCK, H. Recursive and Sequential Tests of the UnitRoot and Trend-Break Hypotheses: Theory and International Evidence. Journal of Business \& Economic Statistics, v. 10, p. 271-287, 1992.

BAXTER, M.; KING, R. G. Measuring Business Cycles: Approximate Band-Pass Filters for Economic Time Series. The Review of Economics and Statistics, v. 81 (4), p. 575-593, 1999.

BEVERIDGE, S.; NELSON, C. R. A. New Approach to Decomposition of Economic Time Series into Permanent and Transitory Components with Particular Attention to Measure- 
ment of the Business Cycle. Journal of Monetary Economics, v. 7, p. 151-174, 1981.

BREITUNG, J.; CANDELON, B. Testing for Short-and Long-Run Causality: A FrequencyDomain Approach. Journal of econometrics, v. 132, n. 2, p. 363-378, 2006.

CARLINO, G.; SILL, K. Regional Income Fluctuations: Common Trends and Common Cycles. The Review of Economics and Statistics, MIT Press, v. 83(3), p. 446-456, 2001.

ENGLE, E. M. R. A. A unified approach to the study of sums, products, time-aggregation and other functions of ARMA processes. Journal of Time Series Analysis, v. 5, p. 159-171, 1984.

ENGLE, R. F.; KOZICKI, S. Testing for Common Features. Journal of Business \& Economic Statistics, v. 11, p. 369-380, 1993.

FARNÉ, M.; MONTANARI, A. A bootstrap test to detect prominent Granger-causalities across frequencies. arXiv preprint arXiv:1803.00374, 2018.

GEWEKE, J. Measurement of linear dependence and feedback between multiple time series. Journal of the American statistical association, v. 77, n. 378, p. 304-313, 1982.

GONZALO, J.; GRANGER, C. W. J. Estimation of Common Long-Memory Components in Cointegrated Systems. Journal of Business and Economics Statistics, v. 33, p. 27-35, 1995.

GUTIERREZ, C. E. C.; GOMES, F. A. R. Evidence on common feature and business cycle synchronization in Mercosur. Brazilian Review of Econometrics, v. 29, p. 37-58, 2009.

HODRICK, R.; PRESCOTT, E. Post-war U.S. Business Cycles: An Empirical Investigation. Working Paper, Carnegie-Mellon, University, 1981. Reimpresso em Journal of Money, Credit and Banking, v. 29, n. 1, 1997.

IBGE. Indicadores Conjunturais da Indústria - Produção. Série Relatórios Metodológicos, v. 31, 2004.

ISSLER, J. V.; VAHID, F. Common cycles and the importance of transitory shocks to macroeconomic aggregates. Journal of Monetary Economics, v. 47, n. 3, p. 449-475, 2001.

JOHANSEN, S. Statistical Analysis of Cointegration Vectors. Journal of Economic Dynamics and Control, v. 12, p. 231-254, 1988.

JOHANSEN, S.; MOSCONI, R.; NIELSEN, B. Cointegration Analysis in the Presence of Structural Breaks in the Deterministic Trend. Econometrics Journal, v. 3, p. 216-249, 2000.

LANNE, M.; LÜTKEPOHL, H.; SAIKKONEN, P. Comparison of unit root tests for time series with level shifts. Journal of time series analysis, v. 23, n. 6, p. 667-685, 2002.

LONG, J. B.; PLOSSER, C. I. Sectoral vs. aggregate shocks in the business cycle. American Economic Review, v. 77, p. 333-336, 1987.

MAYORAL, L. Testing for fractional integration versus short memory with structural breaks. Oxford Bulletin of Economics and Statistics, v. 74, n. 2, p. 278-305, 2012.

MEJÍA-REYES, P.; GÓMEZ, J. A. M.; BALBOA, W. L. Rendón. Ciclos económicos industriales clásicos en México. Colegio Mexiquense, 2004.

MONTEIRO, F. D. S. C.; LIMA, J. P. R. Desindustrialização regional no Brasil. Nova Economia, v. 27, n. 2, 10 out. 2017.

PERRON, P. The Great Crash, the Oil Price Shock, and the Unit Root Hypothesis. Econometrica, v. 57 , p. 1.361-1.401, 1989. 
PERRON, P. Further evidence on breaking trend functions in macroeconomic variables. Journal of econometrics, v. 80, n. 2, p. 355-385, 1997.

PERRON, P.; VOGELSANG, T. J. Nonstationarity and Level Shifts with an Application to Purchasing Power Parity. Journal of Business \& Economic Statistics, v. 10, p. 301-320, 1992.

ROCHA, B. DE P.; SOUZA, I. V. DE. Novas evidências de causalidade entre sistema financeiro e crescimento econômico no Brasil usando séries de tempo no domínio da frequência. Nova Economia, v. 28, n. 1, 4 jun. 2018.

SABOIA, J. A continuidade do processo de desconcentração regional da indústria brasileira nos anos 2000. Nova Economia, v. 23, n. 2, 14 nov. 2013.

WAKERLY, E. C.; SCOTT, B. G.; NASON, J. M. Common Trends and common cycles in Canada: Who knew so much has been going on? Canadian Journal of Economics, v. 39, n. 1, p. 320-347, 2006.

WANG, P. An examination of business cycle features in UK Sectoral Output. Applied Economics, v. 42, n. 25, p. 3.241-3.252, 2010.

WANG, P. Business Cycle Phases and Coherence - a Spectral Analysis of UK Sectoral Output. The Manchester School, v. 81, n. 6, p. 1.012-1.026, 2013.

VAHID, F.; ENGLE, R. F. Common Trends and Common Cycles. Journal of Applied Econometrics, v. 8, p. 341-360, 1993.

VOGELSANG, T. J.; PERRON, P. Additional Test for Unit Root Allowing for a Break in the Trend Function at an Unknown Time. International Economic Review, v. 39, p. 1.073-1.100, 1998.

ZIVOT, E.; ANDREWS, D. W. K. Further Evidence on the Great Crash, the Oil Price Shock and the Unit Root Hypothesis. Journal of Business and Economic Statistics, v. 10, p. 251-270, 1992.

\section{Sobre os autores}

Cristiano da Costa da Silva - cristiano.dacostadasilva@hotmail.com

Universidade do Estado do Rio Grande do Norte, Mossoró, Rio Grande do Norte, Brasil.

ORCID: https://orcid.org/0000-0001-9534-5332.

Nicolino Trompieri Neto-nicolinoneto@gmail.com

Instituto de Pesquisa e Estratégia Econômica do Ceará, Fortaleza, Ceará, Brasil.

Universidade de Fortaleza, Fortaleza, Ceará, Brasil.

ORCID: https://orcid.org/0000-0002-1608-9754.

Ivan Castelar-lume1250@yahoo.com.br

Universidade Federal do Ceará, Fortaleza, Ceará, Brasil.

ORCID: https://orcid.org/0000-0002-7170-1870.

ErikaVanessa Alvesda Silva - erika_vanessa01@hotmail.com

Companhia Pernambucana de Saneamento, Recife, Pernambuco, Brasil.

ORCID: https://orcid.org/0000-0002-6337-2253.

\section{Sobre 0 artigo}

Recebido em 15 de março de 2018. Aprovado em 20 de setembro de 2019 


\section{APÊNDICE}

Tabela A1 Testes de estacionariedade incorporando quebra estrutural séries em $1^{\text {a }}$ diferença

\begin{tabular}{|c|c|c|c|c|c|c|c|c|}
\hline & & BA & MG & $\mathrm{PE}$ & PR & RJ & RS & SP \\
\hline \multirow{2}{*}{$\begin{array}{l}\text { Zivot-Andrews (1992) b. } \\
\text { (HO: Série com R.U.) }\end{array}$} & Teste na $1^{\mathrm{a}}$ & $-5,11$ & $-5,58$ & $-5,86$ & $-5,61$ & $-5,07$ & $-6,01$ & $-8,21$ \\
\hline & Diferença & $* *$ & $* * *$ & $* * *$ & $* * *$ & $* *$ & $* * *$ & $* * *$ \\
\hline \multirow{2}{*}{$\begin{array}{l}\text { Unit Root with Break } \\
\text { Test (1996) c. } \\
\text { (HO: Série com R.U.) }\end{array}$} & Teste na $1^{\mathrm{a}}$ & $-14,30$ & $-12,37$ & $-11,12$ & $-10,17$ & $-17,15$ & $-13,94$ & $-18,68$ \\
\hline & Diferença & $* * *$ & $* * *$ & $* * *$ & $* * *$ & $* * *$ & $* * *$ & $* * *$ \\
\hline \multirow{2}{*}{$\begin{array}{l}\text { Perron (1997) d. } \\
\text { (HO: Série com R.U.) }\end{array}$} & Teste na $1^{\mathrm{a}}$ & $-5,11$ & $-5,58$ & $-5,86$ & $-5,61$ & $-5,07$ & $-6,01$ & $-8,21$ \\
\hline & Diferença & $* *$ & $* * *$ & $* * *$ & $* * *$ & $* *$ & $* * *$ & $* * *$ \\
\hline \multirow{2}{*}{$\begin{array}{l}\text { Lanne et al. e (2002) e } \\
\text { (H0: Série com R.U.) }\end{array}$} & Teste na $1^{\mathrm{a}}$ & $-13,12$ & $-9,87$ & $-11,61$ & $-11,19$ & $-11,57$ & $-10,98$ & $-10,01$ \\
\hline & Diferença & $* * *$ & $* * *$ & $* * *$ & $* * *$ & $* *$ & $* * *$ & $* * *$ \\
\hline
\end{tabular}

Fonte: IBGE.

Estatística treportada. Escolha de defasagens, segundo o critério de Schwarz, com limitação máxima de 12 defasagens * Rejeição da hipótese nula a 10\%, **Rejeição da hipótese nula a 5\%, ***Rejeição da hipótese nula a $1 \%$.

Figura A1 Periodograma das séries temporais - 2012:01/2014:12
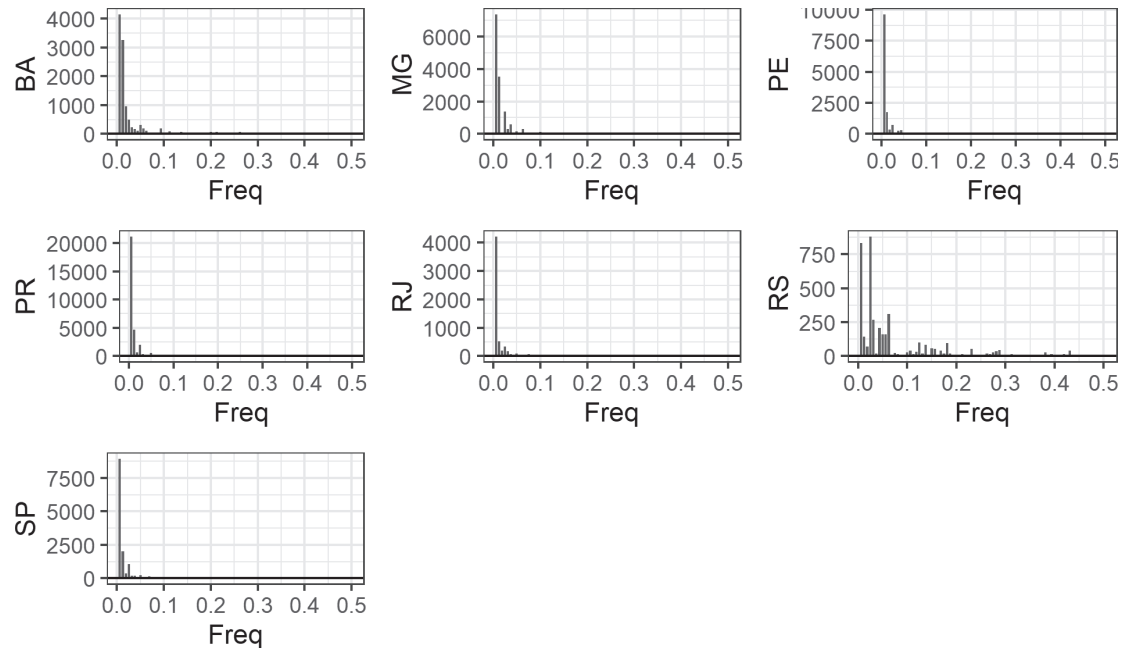

Fonte: Elaborada pelos autores. 
Figura A2 Função autocorrelação das séries temporais - 2012:01/2014:12
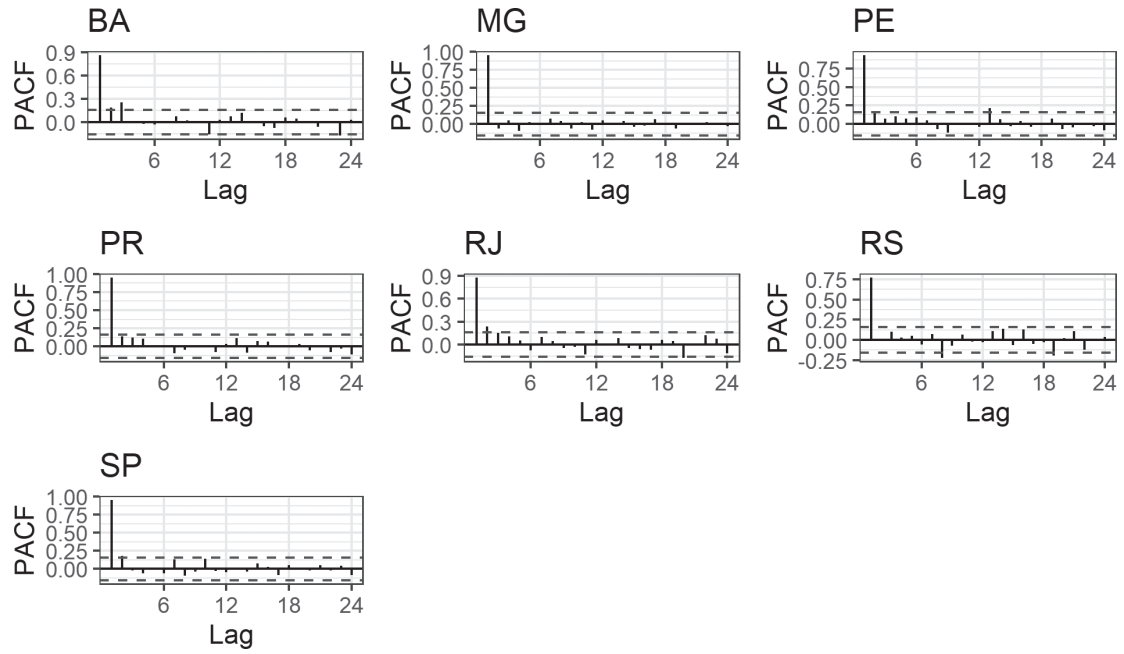

Fonte: Elaborada pelos autores.

Figura A3 Função autocorrelação parcial das séries temporais - 2012:01/2014:12
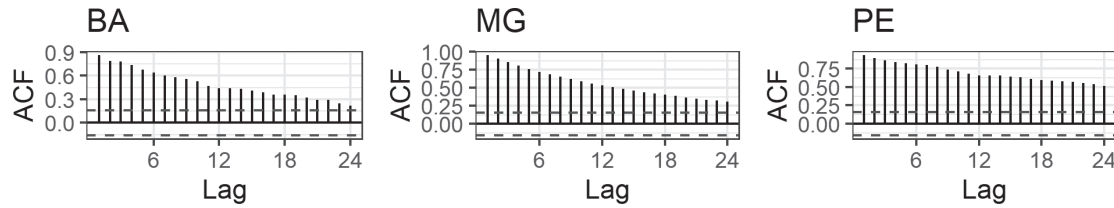

PR

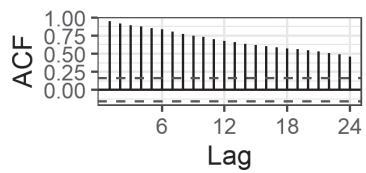

RJ

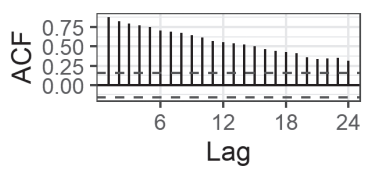

RS
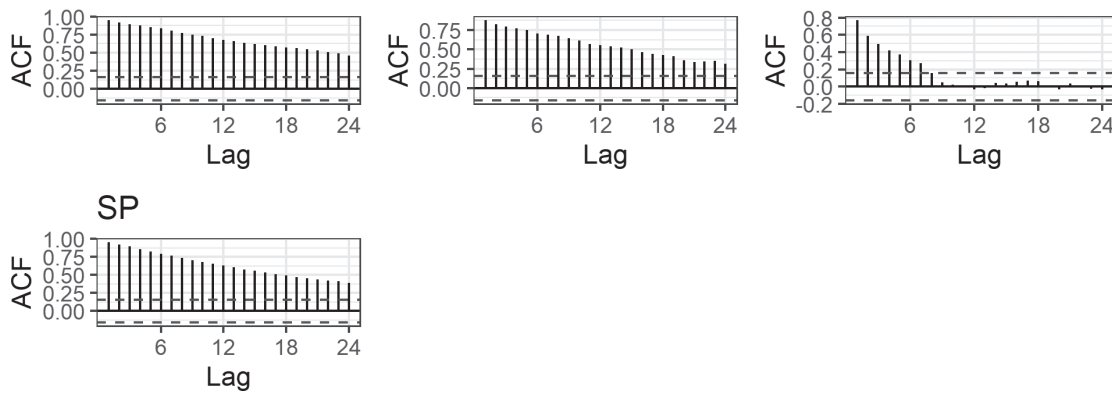

Fonte: Elaborada pelos autores. 